\title{
Understudied G Protein-Coupled Receptors in the Kidney
}

\author{
Nathan A. Zaidman Jennifer L. Pluznick \\ Department of Physiology, Johns Hopkins University School of Medicine, Baltimore, MD, USA
}

\begin{abstract}
Keywords
Kidney - Orphan receptor - Olfactory receptor - Taste receptor $\cdot G$ protein-coupled receptor
\end{abstract}

\begin{abstract}
G protein-coupled receptors (GPCRs) are cell surface proteins which play a key role in allowing cells, tissues, and organs to respond to changes in the external environment in order to maintain homeostasis. Despite the fact that GPCRs are known to play key roles in a variety of tissues, there are a large subset of GPCRs that remain poorly studied. In this minireview, we will summarize what is known regarding the "understudied" GPCRs with respect to renal function, and in so doing will highlight the promise represented by studying this gene family.

๑) 2021 S. Karger AG, Basel
\end{abstract}

\section{Introduction}

Constant surveillance of the external and internal environment is necessary to maintain homeostasis. However, proper monitoring of a huge variety of molecules from simple ions, to hormones and complex metabolites - presents a profound challenge. One of the chief ways this challenge is met is with the use of $\mathrm{G}$ protein-coupled receptors (GPCRs), which comprise the largest superfamily in the genome. GPCRs are cell-surface seven- transmembrane receptors, typically activated by binding to peptides or small molecules. Because GPCRs are expressed in a cell type-specific manner and found on the plasma membrane, GPCRs are promising drug targets: in fact, roughly one-third of FDA-approved drugs target GPCRs.

Despite the promise of GPCRs, to date only a relatively small number of GPCRs in the human genome ( 35\%) have been studied. The remaining GPCRs ("understudied GPCRs") are olfactory receptors $(n=350)$, GPCR taste receptors $(n=28)$, and orphan receptors $(n=140)$. In this minireview, we will review what is known to date regarding the function of these understudied GPCRs with relation to the kidney (Fig. 1).

\section{Olfactory Receptors}

Olfactory receptors are the largest gene family in the genome, and thus, there is great promise in exploring the functional roles of these receptors in the kidney. Canonically, olfactory receptors signal by elevating cAMP; this is

Contribution from the AKI and CRRT 2021 Symposium at the 26th International Conference on Advances in Critical Care Nephrology, A Virtual/Hybrid Event from San Diego, CA, USA, February 28-March 5, 2021. This symposium was supported in part by the NIDDK funded University of Alabama at Birmingham-University of California San Diego O'Brien Center for Acute Kidney Injury Research (P30DK079337).
Correspondence to:

Jennifer L. Pluznick, jpluznick@jhmi.edu 


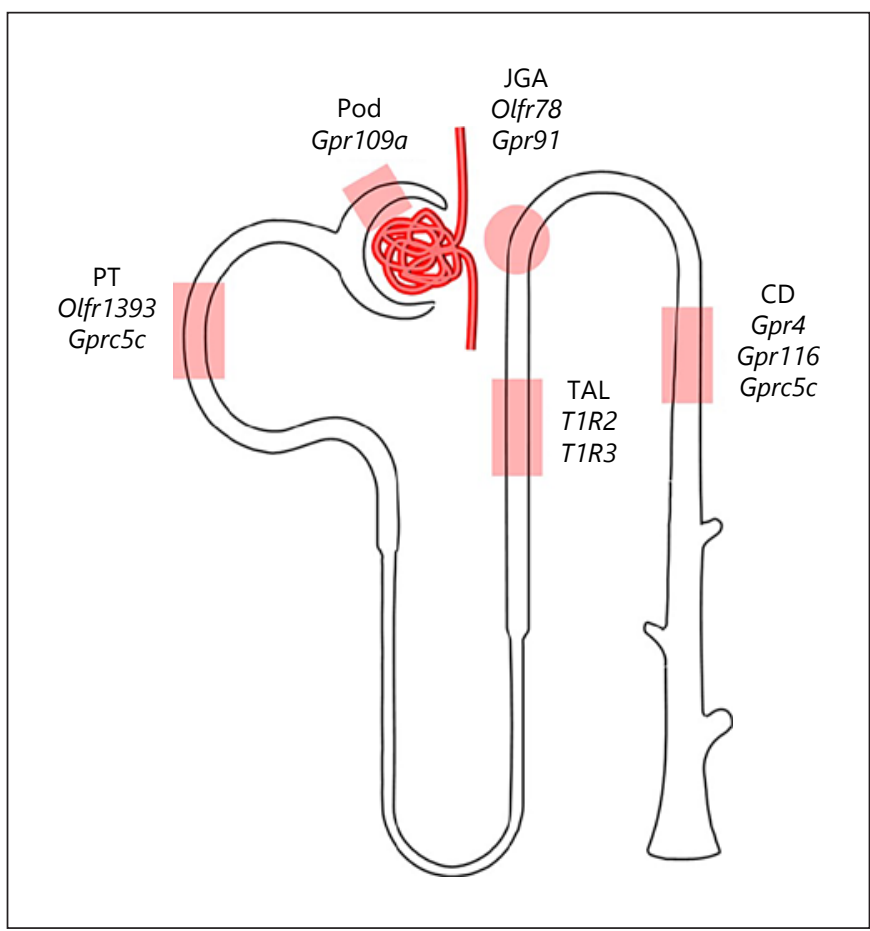

Fig. 1. Localization of understudied GPCRs along the nephron. Pod, podocytes; PT, proximal tubule; TAL, thick ascending limb; $\mathrm{CD}$, collecting duct.

largely assumed to be true of ectopically expressed olfactory receptors as well, although the signaling pathways of olfactory receptors in the kidney are largely unknown. Although multiple studies have reported olfactory receptors expressed in the murine and human kidney [1-3], a much smaller number have outlined specific physiological or pathophysiological roles for these receptors. Key gaps in knowledge which have hindered efforts to uncover physiological roles include an incomplete understanding of the specific cell types of localization of these olfactory receptors, and incomplete (or in some cases, absent) information regarding receptor ligands for this gene family. Nevertheless, functional roles for a few olfactory receptors have been described to date. For example, olfactory receptor 1393 (Olfr1393) was reported to localize to the renal proximal tubule (PT), where it plays a role in regulating renal glucose handling both in health and in disease [4]. Olfr 1393 modulates the apical expression of the $\mathrm{Na}^{+}$/glucose cotransporters Sglt1 and Sglt2, which is particularly notable in that Sglt2 inhibitors are FDA-approved for use in type 2 diabetes.

Another receptor, olfactory receptor 78 (Olfr78), has been reported to localize to blood vessels. This includes the renal afferent arteriole, where Olfr78 plays a role in mediating renin secretion and, consequently, in influencing plasma renin activity [5]. Intriguingly, Olfr78 is activated by short-chain fatty acids (SCFAs) [5], which are produced by the gut microbiota. This implies that renin secretion and blood pressure control are modulated by changes in gut microbial metabolism. Of note, perhaps in part because olfactory receptors are the largest gene family in the genome, identifying orthologs across mammalian species has proven difficult. Thus, it is notable that Olfr78 is one of only 3 olfactory receptors to have a clear ortholog across all placental mammals [6], and it has been demonstrated experimentally that the murine (Olfr78) and human (OR51E2) orthologs have similar ligand profiles [5]. Of note, the ligands that activate Olfr78/OR51E2 have been implicated as having beneficial effects in acute kidney injury, and it is possible that the mechanisms involved include activation of Olfr78/OR51E2 and other, similar, nonolfactory receptors which respond to SCFAs [7]; future studies will be needed to determine the role of each SCFA receptor in protection in acute kidney injury.

Of note, there are a number of other olfactory receptors which have been identified as expressed in the kidney [1-3] but which have not yet been studied; in the future, it will be important to expand our knowledge of olfactory receptors in the kidney to include these unstudied receptors. In addition, our knowledge of downstream signaling pathways for Olfr1393 and Olfr78 is quite limited; this is another area that will be important to pursue in future studies.

\section{Taste Receptors}

Although taste receptors have been reported in the kidney $[1,8]$, few studies to date have outlined their roles in renal function. Thus, this is clearly an area in acute need of future study. Liu et al. [9] identified the bitter taste receptor Tas2r105 in the kidney and suggested it helps maintain the structure of the glomeruli/tubules. Again, it is clear that this is an area ripe for discovery, and future work will be critical in revealing the functional roles of taste receptors in renal function.

\section{GPR Receptors}

GPCRs without ligand partners are often called orphan receptors. Many of these GPCRs carry names with the prefix "Gpr," reflecting a common lack of information 
when these receptors were identified. Significant progress has been made in the past decade to deorphanize these GPCRs. Central to this review, 6 GPRs were previously identified as being strongly expressed in whole murine kidney tissue: Gprc5c, Gpr56, Gpr116, Gpr137, Gpr48, and Gpr 137b [1]. Studies performed on Gprc5c identified the receptor as a regulator of NHE3 activity in the PT [10]. Rajkumar et al. [10] revealed that Gprc5c localizes to the apical membrane of the PT and increases NHE3 activity at alkaline extracellular $\mathrm{pH}$ in heterologous expression studies. Notably, Gprc5c knock-out mice excrete alkaline urine compared to WT littermates and have reduced NHE3 activity along the PT.

More recently, studies have also revealed a significant role for Gpr116 in renal physiology [11]. Similar to Gprc5c, Gpr116 regulates renal acid-base handling. Localized to A-type intercalated cells in the murine collecting ducts, tubule-specific Gpr116 knock-out mice excrete acidic urine and have slightly alkaline blood compared to WT littermates. Additionally, Zaidman et al. [11] demonstrated that Gpr116 KO animals have physiologically inappropriate distribution of $\mathrm{V}$-ATPase proton pumps on the surface of A-intercalated cells, revealing the molecular mechanism by which Gpr116 regulates urine $\mathrm{pH}$. In fact, activation of Gpr 116 with a synthetic agonist peptide significantly inhibits $\mathrm{pH}_{\mathrm{i}}$ recovery in A-type cells, further demonstrating that Gpr116 negatively regulates VATPase surface expression.

Additionally, previous work has uncovered significant physiological roles for other GPRs in the kidney. For example, genetic deletion of Gpr4, a pH-sensitive GPCR expressed in the collecting ducts, reduces net acid secretion, thereby leading to acidosis [12]. Furthermore, Gpr4 knockout reduces renal injury caused by ischemia-reperfusion by reducing apoptosis [13]. The succinate receptor Gpr91 was identified as a mediator of glucose-stimulated renin release in the juxtaglomerular apparatus [14]. Lastly, Gpr109a, a butyrate-sensing receptor expressed in podocytes, protects against adriamycin-induced (ADR) nephropathy by preserving the glomerular basement membrane [15]. In this study, mice fed a prebiotic diet leading to high concentrations of butyrate were protected from ADR nephropathy in part through TsA-sensitive epigenetic effects, whereas Gpr109a knockout mice gained no protection against ADR nephropathy with increased butyrate. Of note, there are a number of orphan receptors/GPRs identified in the kidney which have not yet been studied, and in future studies, it will be crucial to uncover the physiological and pathophysiological roles of these receptors.

\section{Conclusion}

As highlighted above, significant progress has been achieved in identifying and studying previously understudied GPCRs in the kidney. Although there still remains much to learn about the renal repertoire of GPCRs, the pioneering discoveries outlined in this short review have generated blueprints for future research and should provide strong assurance to the benefits of undertaking such open-ended projects.

\section{Conflict of Interest Statement}

The authors declare that there are no conflicts of interest.

\section{Funding Sources}

This work was supported by F32DK116499 (to N.A.Z.), R01DK107726 (to J.L.P.), and R01HL128512 (to J.L.P.).

\section{Author Contributions}

N.A.Z. and J.L.P. both wrote and edited this manuscript and approved the final version.

\section{References}

1 Rajkumar P, Aisenberg WH, Acres OW, Protzko RJ, Pluznick JL. Identification and characterization of novel renal sensory receptors. PLoS One. 2014;9:e111053.

2 Halperin Kuhns VL, Sanchez J, Sarver DC, Khalil Z, Rajkumar P, Marr KA, et al. Characterizing novel olfactory receptors expressed in the murine renal cortex. Am J Physiol Renal Physiol. 2019;317:F172-86.
3 Kalbe B, Schlimm M, Wojcik S, Philippou S, Massberg D, Jansen F, et al. Olfactory signaling components and olfactory receptors are expressed in tubule cells of the human kidney. Arch Biochem Biophys. 2016;610:8-15.

4 Shepard BD, Koepsell H, Pluznick JL. Renal olfactory receptor 1393 contributes to the progression of type 2 diabetes in a diet-induced obesity model. Am J Physiol Renal Physiol. 2019;316:F372-81. 
5 Pluznick JL, Protzko RJ, Gevorgyan H, Peterlin Z, Sipos A, Han J, et al. Olfactory receptor responding to gut microbiota-derived signals plays a role in renin secretion and blood pressure regulation. Proc Natl Acad Sci U S A. 2013;110:4410-5.

6 Niimura Y, Matsui A, Touhara K. Extreme expansion of the olfactory receptor gene repertoire in african elephants and evolutionary dynamics of orthologous gene groups in 13 placental mammals. Genome Res. 2014;24: 1485-96.

7 Gong J, Noel S, Pluznick JL, Hamad ARA, Rabb H. Gut microbiota-kidney cross-talk in acute kidney injury. Semin Nephrol. 2019; 39(1):107-16.

8 Liang J, Chen F, Gu F, Liu X, Li F, Du D. Expression and functional activity of bitter taste receptors in primary renal tubular epithelial cells and m-1 cells. Mol Cell Biochem. 2017; 428:193-202.

9 Liu X, Gu F, Jiang L, Chen F, Li F. Expression of bitter taste receptor tas2r105 in mouse kidney. Biochem Biophys Res Commun. 2015; 458:733-8.

10 Rajkumar P, Cha B, Yin J, Arend LJ, Păunescu TG, Hirabayashi Y, et al. Identifying the localization and exploring a functional role for gprc5 $\mathrm{c}$ in the kidney. FASEB J. 2018;32:2046-59.

11 Zaidman NA, Tomilin VN, Hassanzadeh Khayyat N, Damarla M, Tidmore J, Capen DE, et al. Adhesion-gpcr gpr116 (adgrf5) expression inhibits renal acid secretion. Proc Natl Acad Sci U S A. 2020.

12 Sun X, Yang LV, Tiegs BC, Arend LJ, McGraw DW, Penn RB, et al. Deletion of the ph sensor gpr4 decreases renal acid excretion. J Am Soc Nephrol. 2010;21:1745-55.

13 Dong B, Zhang X, Fan Y, Cao S, Zhang X. Gpr4 knockout improves renal ischemia-reperfusion injury and inhibits apoptosis via suppressing the expression of chop. Biochem J. 2017;474:4065-74.

14 Toma I, Kang JJ, Sipos A, Vargas S, Bansal E, Hanner F, et al. Succinate receptor gpr91 provides a direct link between high glucose levels and renin release in murine and rabbit kidney. J Clin Invest. 2008;118:2526-34.

15 Felizardo RJF, de Almeida DC, Pereira RL, Watanabe IKM, Doimo NTS, Ribeiro WR, et al. Gut microbial metabolite butyrate protects against proteinuric kidney disease through epigenetic- and gpr109a-mediated mechanisms. FASEB J. 2019;33:11894-908. 